\title{
Pratiques Paysannes De Production Durable Des Graines De Voandzou [Vigna Subterranea (L.) Verdc.] Pour La Sécurité Alimentaire Dans Le Cameroun Septentrional
}

\section{Madou Chantal,}

Université de Ngaoundéré, Faculté des Sciences, Département des Sciences Biologiques, Ngaoundéré Cameroun. Institut de Recherche Agricole pour le

Développement (IRAD), Station Polyvalente de Recherche de Garoua, Garoua Cameroun.

\section{Watching Djakissam, Vatsou Jeremie, Ardjoune Fatime,}

Université de Ngaoundéré, Faculté des Sciences,

Département des Sciences Biologiques, Ngaoundéré Cameroun.

Ndjouenkeu Robert,

Université de Ngaoundéré, Ecole Nationale Supérieure des Sciences Agro Industrielles, Département de Chimie Appliquée, Ngaoundéré Cameroun.

\section{Goudoum Augustin,}

Université de Maroua, Ecole Nationale Supérieure Polytechnique de Maroua, Département d'Agriculture, Elevage et Produits Dérivés,

Maroua, Cameroun.

\section{Ngassoum Martin Benoit,}

Université de Ngaoundéré, Ecole Nationale Supérieure des Sciences Agro Industrielles, Département de Chimie Appliquée, Ngaoundéré Cameroun.

\section{Ngamo Tinkeu Simon Leonard,}

Université de Ngaoundéré, Faculté des Sciences, Département des Sciences

Biologiques, Ngaoundéré Cameroun.

Doi: 10.19044/esj.2018.v14n18p424 URL:http://dx.doi.org/10.19044/esj.2018.v14n18p424

\section{Abstract}

Bambara groundnut is a leguminous plant with high nutritive value of Sub Saharan Africa. In spite of its high potential in alimentation, the Bambara groundnut is less popularized. This crop grows on acid, dry and less fertile soils and may therefore be a resilient crop in the context of climatic variability. The present work aims to identify of all the morphotypes of this culture and 
the usual tools of conservation of its seeds during the storage process. Investigations carried out during 3 agricultural campaigns showed that women more than 40 years old represents more than $98 \%$ of producers of Bambara groundnuts in northern Cameroon. The production concerns 54 morphotypes use as food or as medicinal plants. During storage of grains of this legumes, the major pest is the bruchid Callosobruchus maculatus Fab. (Coleoptera : Chrysomelidae). Under traditional production way, the limitation of the postharvest losses due to this pest is done either by the use of 21 local plants as botanical insecticide or by the use of 25 industrial chemical pesticides.

Keywords: Bambara groundnut, insect pest, stored product, botanical insecticide, Cameroon

\section{Resume}

Le voandzou, encore appelé pois Bambara ou pois de terre, est une légumineuse alimentaire cultivée en Afrique sub-saharienne. En dépit de sa très forte valeur alimentaire cette légumineuse n'est pas vulgarisée. Sa tolérance pour les sols secs, acides et peu fertiles fait d'elle une culture de résilience pour l'adaptation à la variabilité climatique. L'objectif de cette recherche est d'identifier tous ses morphotypes et les outils usuels de conservation de ses graines au cours du stockage. Au terme de trois campagnes de collecte de données de terrain, il est clair que $98 \%$ des producteurs de voandzou sont des femmes. Parmi elles $60 \%$ sont âgées de plus de 40 ans. La production concerne 54 morphotypes qui sont utilisés dans l'alimentation et dans la pharmacopée. Callosobruchus maculatus Fab. (Coleoptera: Chrysomelidae) est le principal ravageur du voandzou. En milieu traditionnel, la limitation des pertes post-récolte dues à ce ravageur se fait soit par usage de 21 plantes locales à vertu insecticide soit par utilisation de près de 25 pesticides chimiques industriels.

Mots clés : Voandzou, ravageurs, denrées stockées, phyto insecticides, Cameroun

\section{Introduction}

Pour accroître durablement sa productivité agricole face aux défis de la variabilité climatique, facteur aggravant de la pauvreté et de l'insécurité alimentaire, le continent africain doit trouver des solutions endogènes. C'est particulièrement le cas pour les petits producteurs pour lesquels le choix des cultures est un facteur déterminant pour assurer la sécurité alimentaire. Les légumineuses sont des sources de protéines végétales facilement accessibles aux populations. En zone tropicale, le niébé (Vigna unguiculata L. Walp.), l'arachide (Arachis hypogeae L.) sont largement consommées contrairement 
à d'autres espèces comme le voandzou (Yusuf et al., 2008). Pourtant, le voandzou [Vigna subterranea (L.) Verdc.] est une espèce particulièrement intéressante car elle supporte l'irrégularité des pluies, pousse en contre saison et tolère un déficit hydrique important. De plus, elle supporte des sols acides et pauvres entre autres contraintes (Pasquet \& Fotso 1991). Le voandzou, encore appelé pois Bambara ou pois de terre, est une légumineuse alimentaire mineure dont la culture est restée dans les limites de l'Afrique sub-saharienne où elle est originaire et est adaptée aux conditions climatiques et écologiques de steppe, de savane et de forêt (Onwubiko et al., 2011). Le voandzou est moins exigeant en eau et se développe facilement sur des terres marginales où il donne des revenus substantiels. Il s'agit donc d'un bon choix résilient, qui en plus a une forte valeur nutritive et un potentiel pour la lutte contre la faim. Ainsi, de toutes les légumineuses alimentaires, le voandzou est la plus riche en méthionine, et en lysine (Stephens, 2009), bien qu'elle n'ait pas suscité beaucoup d'intérêt pour la recherche et le développement.

Les graines seules ou mélangées à d'autres aliments servent à l'alimentation humaine et en médecine traditionnelle alors que les feuilles, riches en phosphore, servent à l'alimentation du bétail (Nacoulma-Ouédraogo, 1996). Dans le Nord-Cameroun, où les céréales et les légumineuses constituent la base de la nourriture des populations, le voandzou est encore une des légumineuses les plus négligées quoique cette culture dite de la femme soit hautement énergétique (Ngamo et al., 2016). Malgré cette importance alimentaire, cette culture n'est apparue dans les statistiques agricoles du Cameroun qu'en 1998 et jusqu'en 2013, la production nationale ne dépassait pas encore les 40 tonnes/an (FAO 2017). L'amélioration de la visibilité et de la disponibilité de cette culture exige une bonne connaissance de la diversité et des performances de chaque cultivar.

La présente étude se donne pour objectif général d'identifier tous les morphotypes de voandzou cultivés par les paysans des régions septentrionales du Cameroun et les techniques de protection post récolte efficaces qui préservent la qualité et la quantité des graines au terme du stockage. Plus spécifiquement, des prospections ont été réalisées auprès des producteurs dans les Régions de l'Adamaoua, du Nord et de l'Extrême-Nord Cameroun, afin de fournir des informations sur les systèmes de production et de stockage du voandzou dans le but de décrire le circuit de cette denrée. De plus, la diversité des morphotypes par bassin de production, les usages, les quantités cultivées, les formes sous lesquelles le voandzou est stocké, les ravageurs, les modules de stockage et les méthodes de conservation les plus fréquentes chez les producteurs ont été répertoriés. A l'issue de ces enquêtes, la consolidation de toutes ces informations facilitera la mise à disposition des données fiables et l'introduction du voandzou dans les programmes de relèvement de la dénutrition pour la promotion de la sécurité alimentaire. 


\section{Materiel et methodes}

\section{Présentation de la zone d'étude}

Sur le continent africain, le Cameroun est logé au fond du Golfe de Guinée et se trouve en position charnière entre l'Afrique occidentale et 1'Afrique Centrale. Le Cameroun s'étale du Sud au Nord entre $1^{\circ} 40$ de latitude Nord et $13^{\circ}$ de latitude Nord sur environ $1250 \mathrm{~km}$. De 1'Est en Ouest, il s'étire entre $8^{\circ} 30$ de longitude Est et $16^{\circ} 10$ de longitude Est, sur environ $860 \mathrm{~km}$. L'espace d'étude se situe entre le $6^{\circ}$ et le $13^{\circ}$ latitude Nord et entre le $11^{\circ}$ et le $15^{\circ}$ parallèle longitude Est. Le climat est à dominance tropical sahélien. Dans l'Adamaoua, il y a deux saisons (humide et sèche) et plus au Nord le climat est de type tropical sec caractérisé par au moins sept mois de sécheresse et des précipitations annuelles variant de 1600 à $500 \mathrm{~mm}$. La Période de Croissance Végétale (PCV) varie du fait de ces pluies. Dans la zone des hautes savanes guinéennes (Adamaoua) où il pleut plus longtemps, cette période est plus longue et peut atteindre 250 jours alors que dans les zones sahéliennes plus sèche (extrême-Nord), certaines années, elle atteint difficilement 100 jours. La température moyenne annuelle atteint $28^{\circ} \mathrm{C}$ avec une amplitude de 7,7 ${ }^{\circ} \mathrm{C}$ (Manetsa, 2011).

L'organisation administrative divise chaque Région en unités administratives que sont les trois Régions du Cameroun septentrional. Elles sont subdivisées en 15 Départements. Dans chaque Département, dix localités ont été visitées par rapport à la disponibilité des graines de voandzou sur les marchés périodiques et chez les cultivateurs, dans leurs réserves familiales. Ces visites ont été effectuées au cours de trois campagnes agricoles successives : en mars et avril pendant la campagne agricole 2014/2015, en Avril pendant la campagne agricole 2015/2016 et en Juin pendant la campagne agricole 2016/2017. Au cours de la prospection dans chaque localité, une fiche d'enquête permettant la collecte des informations sur la gestion des graines au cours du stockage est utilisée et deux catégories de cibles visées : les vendeurs et les producteurs de voandzou.

\section{Méthodologie de la collecte des données et des échantillons de voandzou}

Dans toutes les localités visitées au moins dix acteurs ont été interviewés. Les interviews ont permis de collecter des informations sur la diversité et les quantités de graines de voandzou récoltées et stockées; la forme sous laquelle la denrée est stockée, le type de module de stockage, la durée de stockage, les ravageurs potentiels et la méthode de protection la plus efficace. A la fin de l'interview, chez les producteurs qui avaient des stocks de voandzou, un prélèvement était réalisé. Les grains ont été aussi achetés sur les marchés locaux. Le choix des graines à acquérir était orienté vers les lots avec plus de diversité de formes et de couleurs de graines de façon à collecter le plus grand nombre possible de morphotypes. Que l'interview ait eu lieu au 
marché ou dans la case familiale, l'entretien a consisté en un entretien direct, où les questions étaient ouvertes ou fermées, en fonction de la précision recherchée sur une question donnée.

\section{Méthode de description des écotypes de voandzou}

Les graines de voandzou collectées au cours de la prospection ont été décrites. Cette caractérisation s'est faite sur la base des descripteurs tels que : la coloration du tégument, la taille des graines et le poids de 50 graines, le contour du hile, son aspect, sa forme et le nombre des graines par gousse. Le poids de 50 graines enregistré est la moyenne de quatre pesées de cinquante graines de chaque morphotype à l'aide d'une balance de précision de marque PRL-4200 FF. Suivant les indications de la liste des descripteurs du voandzou (IPGRI/IITA/BAMNET, 2000)

\section{Diversité de l'entomofaune des graines de voandzou au cours du stockage et outils usuels de limitation des pertes post-récoltes}

Les graines de voandzou collectées ont été observées dans les pots en laboratoire et l'émergence des bruches ou tout autre insecte inféodé aux denrées stockées a été noté. Ces insectes ont été identifiés grâce à la clé de reconnaissance des insectes ravageurs des denrées stockées en milieu tropical chaud de Delobel et Tran (1991). Les outils de protection de ces denrées que les producteurs utilisent ont été identifiés. Pour les pesticides chimiques industriels, la référence est 1'Index Phytosanitaire du Cameroun (MINADER, 2014). Les plantes actives introduites dans les greniers au moment du stockage pour éloigner les ravageurs, ont été prélevées quand les producteurs pouvaient les reconnaitre. Elles ont été par la suite mises en herbier et identifiées. L'identification s'est faite d'après un herbier de référence constitué par Malaisse F., Professeur émérite de botanique à la FSAGx, Belgique et Mapongmetsem P-M, ethno botaniste, Professeur titulaire de botanique à la Faculté des Sciences de 1'Université de Ngaoundéré.

\section{Resultats et discussion}

Les producteurs, la production et la diversité des morphotypes et les usages des graines de voandzou

Les données collectées au cours des trois campagnes agricoles, ont été régulières pour 15 départements des trois régions de l'espace d'étude. Soit un total de 643 personnes interrogé sur la base d'un formulaire. Avec respectivement 151 dans l'Adamaoua, 159 dans le Nord et 333 dans 1'Extrême-Nord (Figure 2). La culture du voandzou de moindre intérêt n'occupe que les femmes qui s'y consacrent en dehors des activités les plus importantes pour la famille. Le voandzou est de ce fait l'une des principales sources de revenus des femmes qui sont les seules à mener cette activité et 
peuvent jouir du revenu de cette activité. Les productrices du voandzou sont en général âgées de plus de 40 ans (figure 1) pour environ $60 \%$ des productrices enquêtées.

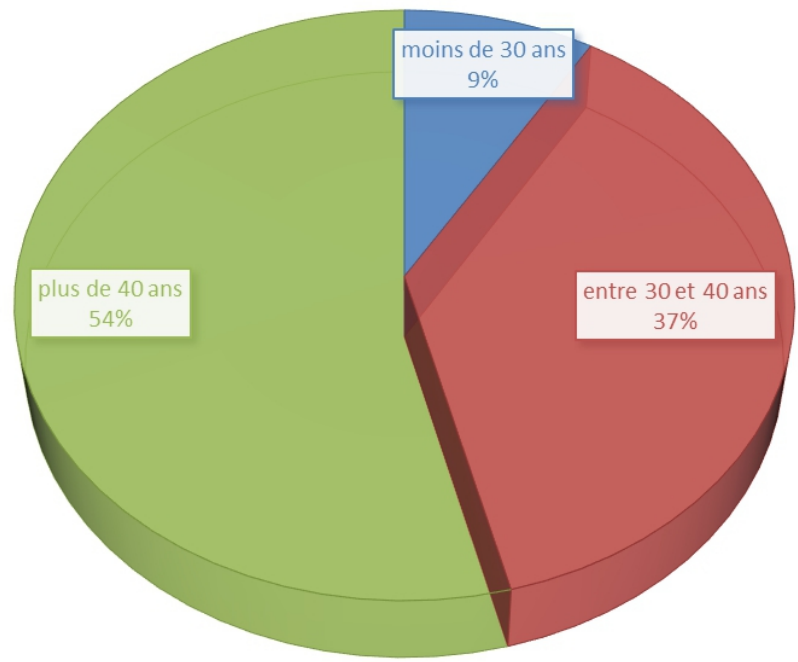

Figure 1: Structure d'âge des 626 cultivatrices du voandzou dans le Cameroun septentrional pendant trois campagnes agricoles de 2014/2015 à 2016/2017

\section{Production du voandzou}

Les graines après la récolte sont stockées en gousse avec leur coque ou nues. La conservation se fait dans des sacs de $80 \mathrm{Kgs}$ environ où les graines sont mises sans aucun tri préalable. Les productions varient, l'Extrême-Nord est la région où les productions cumulées sur les trois années sont les plus importantes avec 340 sacs, suivie de la région du Nord (255 sacs) et enfin de celle de l'Adamaoua où les productions sont les plus faibles avec 164 sacs (Tableau 1).

Tableau 1. Les producteurs du voandzou rencontrés (H : Homme ; F : Femme), diversité des morphotypes (Mo) et importance de la production moyenne dans les trois régions septentrionales du Cameroun au cours des campagnes agricoles 2014/2015 à 2016/2017.

\begin{tabular}{llll}
\hline Région & Département (Chef-lieu) & $\begin{array}{l}\text { Producteurs } \\
(\mathrm{F} / \mathrm{H})\end{array}$ & $\begin{array}{l}\text { Production* } \\
(\mathrm{Mo}) / \mathrm{Morp}\end{array}$ \\
\hline Adamaoua & Vina (Ngaoundéré) & $30 / 1$ & $3,41(4)$ \\
$(\mathrm{n}=151 ;$ & Mbéré (Meiganga) & $27 / 2$ & $2,77(5)$ \\
Femmes $=$ & Mayo Banyo (Banyo) & $31 / 0$ & $2,09(3)$ \\
$97,35 \%)$ & Faro \& Déo (Tignère) & $18 / 0$ & $1(4)$ \\
& Djérem (Tibati) & $41 / 1$ & $2,93(4)$ \\
\hline Nord & Bénoué (Garoua) & $74 / 2$ & $4,11(6)$ \\
$(\mathrm{n}=159 ;$ & Faro (Poli) & $24 / 0$ & $3,93(4)$ \\
Femmes $=$ & Mayo Louti (Guider) & $30 / 0$ & $2(3)$ \\
$98,11 \%)$ & Mayo Rey (Tcholiré) & $28 / 1$ & $2,78(8)$ \\
\hline Extrême Nord & Diamaré (Maroua) & $79 / 3$ & $3,68(5)$
\end{tabular}




\begin{tabular}{llll}
\hline$(\mathrm{n}=333 ;$ & Mayo Kani (Kaélé) & $99 / 1$ & $3,96(12)$ \\
Femmes $=$ & Mayo Danay (Yagoua) & $53 / 2$ & $4,28(8)$ \\
$97,06 \%)$ & $\begin{array}{l}\text { Mayo Tsanaga } \\
\text { (Mokolo) }\end{array}$ & $36 / 0$ & $2,72(6)$ \\
& $\begin{array}{l}\text { Logone et chari } \\
\text { (Kousséri) }\end{array}$ & $24 / 1$ & $2,95(7)$ \\
& Mayo Sava (Mora) & & $2,35(7)$ \\
Total (643) & & $626 / 17$ &
\end{tabular}

\section{Diversité de morphotypes et préférences des cultivatrices}

Dans les régions septentrionales du Cameroun, la culture du voandzou est largement connue mais peu pratiquée. Les surfaces cultivées sont réduites soit $1 / 4$ ha et les productions faibles 3 à 5 sacs de $100 \mathrm{Kg}$. Par ailleurs, au sein de ces quantités cultivées, il existe également une grande variabilité phénotypique basée sur la coloration du tégument et sur la morphologie de la graine (Tableau 2). Bien que situées dans la même zone agro-écologique, certains morphotypes sont spécifiques à certaines régions et d'autres distribués aléatoirement dans l'ensemble des trois zones de production. En somme, 54 morphotypes sont recensés dans la zone d'étude (Figure 2). 


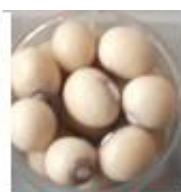

$\mathrm{CM} / \mathrm{EN} / \mathrm{M} / \operatorname{or}^{-}$

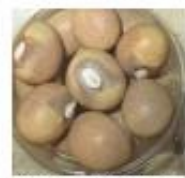

$\mathrm{CM} / \mathrm{AD} / \mathrm{MC} / 29$

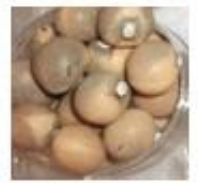

$\mathrm{CM} / \mathrm{AD} / \mathrm{MC} / \propto 6$
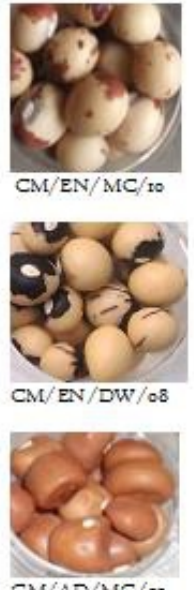

$\mathrm{CM} / \mathrm{AD} / \mathrm{MC} / \mathrm{og}$

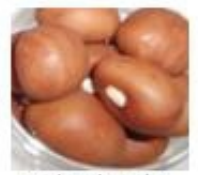

$\mathrm{CM} / \mathrm{EN} / \mathrm{MC} / \mathrm{r} 8$
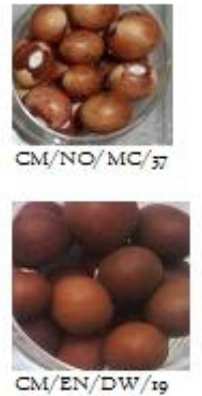

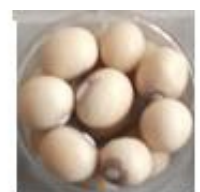

$\mathrm{CM} / \mathrm{EN} / \mathrm{AE} / \mathrm{oz}$

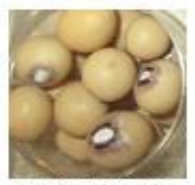

$\mathrm{CM} / \mathrm{AD} / \mathrm{DW} / 32$

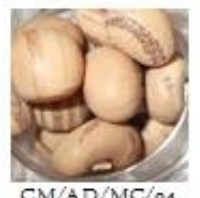

$\mathrm{CM} / \mathrm{AD} / \mathrm{MC} / 04$
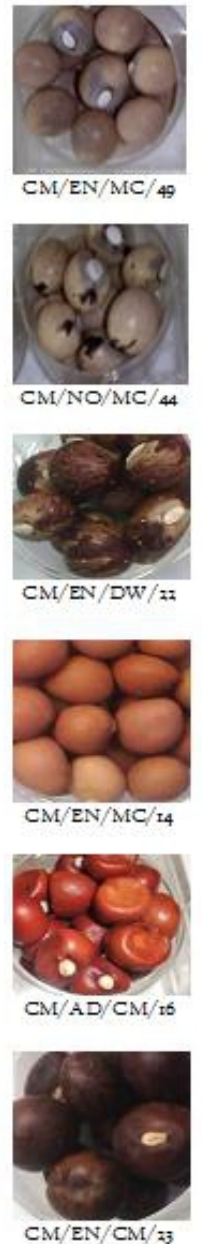

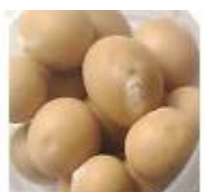

$\mathrm{CM} / \mathrm{EN} / \mathrm{DW} /$ or
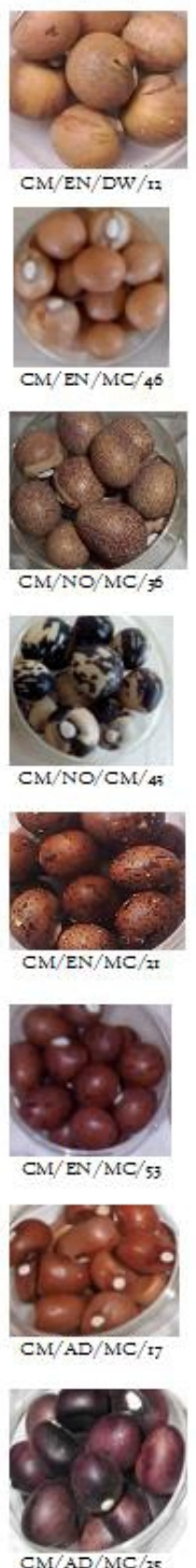
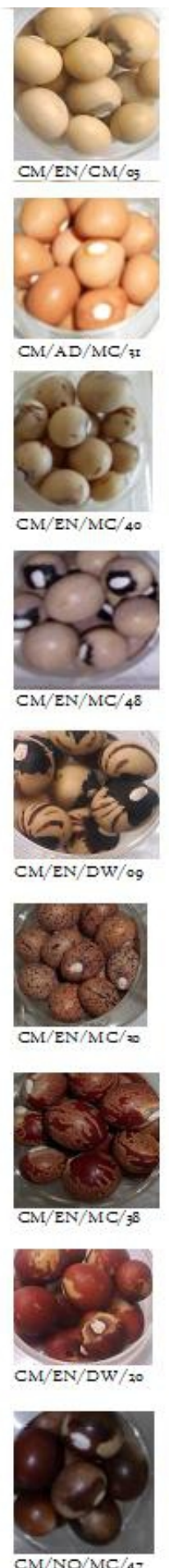

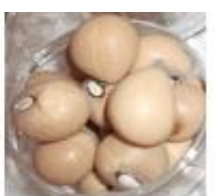

$\mathrm{CM} / \mathrm{AD} / \mathrm{MC} / 05$

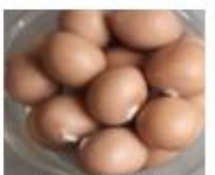

$\mathrm{CM} / \mathrm{AD} / \mathrm{MC} / \mathrm{r}$
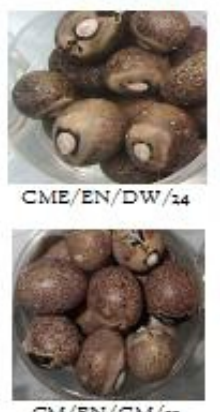

$\mathrm{CM} / \mathrm{EN} / \mathrm{CM} / 39$
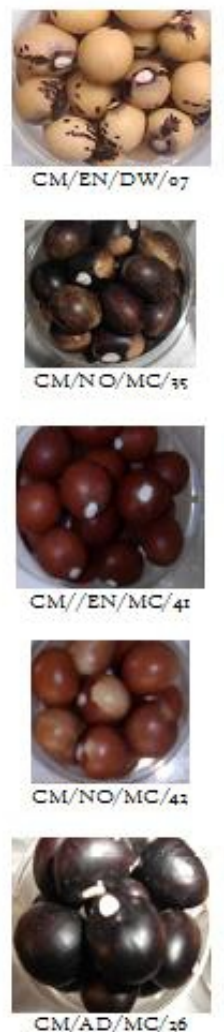

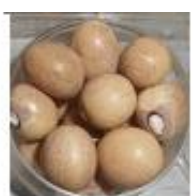

$\mathrm{CM} / \mathrm{EN} / \mathrm{MC} / 34$
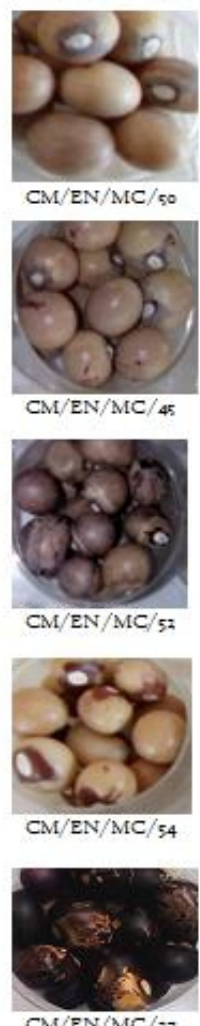

$\mathrm{CM} / \mathrm{EN} / \mathrm{MC} / 27$
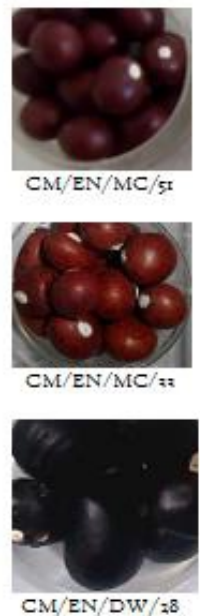

Figure 2 : Illustration des 54 morphotypes de voandzou collectés dans le Cameroun septentrional au cours des campagnes agricoles 2014/2015 à 2016/2017. 
Tableau 2 : Description de la diversité des morphotypes de voandzou identifiés dans les trois régions septentrionales du Cameroun au cours des trois campagnes agricoles ciblées

\begin{tabular}{|c|c|c|c|c|c|c|c|}
\hline code & Couleur du tégument & $\begin{array}{l}\text { Forme de la } \\
\text { graine }\end{array}$ & $\begin{array}{l}\text { Poids 50 } \\
\text { graine (g) }\end{array}$ & $\begin{array}{c}\text { Aspect du hile de la } \\
\text { graine }\end{array}$ & $\begin{array}{l}\text { Graine/ } \\
\text { gousse }\end{array}$ & Ravageur* & lieu de collecte \\
\hline CM/EN/DW/01 & Blanc laiteux & Ronde & 55 & Sans contour & une & C. $m$ & Gazawa, Mokolo, Mindif, Poli \\
\hline CM/EN/AE/02 & Blanc crème & Ronde & 45 & $\begin{array}{l}\text { Contour violet en } \\
\text { forme de papillon }\end{array}$ & une & C. $m$ & $\begin{array}{l}\text { Dziguilao, Guidiguis, Yagoua, } \\
\text { Salak, Guider, Lagdo, Gazawa }\end{array}$ \\
\hline $\mathrm{CM} / \mathrm{EN} / \mathrm{DW} / 03$ & Blanc laiteux & Ronde & 42 & $\begin{array}{l}\text { Contour violet en } \\
\text { forme de papillon }\end{array}$ & une & C. $m$ & Bogo, Guidiguis, Mindif, Yagoua \\
\hline $\mathrm{CM} / \mathrm{AD} / \mathrm{MC} / 04$ & Beige rayure gris & ovale & 50,25 & Sans contour & une & $C . m$ & Kaélé, Galim-tignère \\
\hline $\mathrm{CM} / \mathrm{AD} / \mathrm{CM} / 05$ & beige & ovale & 50.10 & noir et blanc & une & C. $s$ & Galim-tignere \\
\hline $\mathrm{CM} / \mathrm{AD} / \mathrm{CM} / 06$ & Tache verte sur fond blanc & ovale & 50 & $\begin{array}{l}\text { Vert en forme } \\
\text { papillon }\end{array}$ & une & C. $s$ & Galim-tignere \\
\hline $\mathrm{CM} / \mathrm{EN} / \mathrm{DW} / 07$ & Rayure noir sur fond blanc & Ronde & 29.5 & $\begin{array}{l}\text { Noir en forme } \\
\text { papillon }\end{array}$ & une & $C . m$ & Mokolo, Dimeo \\
\hline $\mathrm{CM} / \mathrm{EN} / \mathrm{DW} / 08$ & Rayure violet sur fond blanc & Ronde & 30 & $\begin{array}{l}\text { Contour noir } \\
\text { irrégulier }\end{array}$ & une & C. $m$ & Moutourwa \\
\hline CM/EN/DW/09 & Blanc avec grosse rayure violet & ovale & 58.5 & $\begin{array}{l}\text { noir en forme } \\
\text { circulaire }\end{array}$ & une & C. $m / C . s$ & $\begin{array}{c}\text { Mora, Moutourwa, Kalfou, } \\
\text { Kolofata }\end{array}$ \\
\hline CM/EN/AE/10 & Blanc tacheté de rouge & Ronde & 36 & contour blanc & une & C. $m$ & Kolofata, Mora \\
\hline $\mathrm{CM} / \mathrm{AD} / \mathrm{CM} / 11$ & Blanc pointillé de violet & ovale & 45 & noire et blanc & une & C. $s$ & Galim -tignere \\
\hline CM/EN/DW/12 & Gris et rayure marron & ovale & 80 & Sans contour du hile & une & C.m & Kaélé, Lara, Ngong, Figuil \\
\hline $\mathrm{CM} / \mathrm{EN} / \mathrm{AE} / 13$ & Gris claire & ovale & 43 & Sans contour du hile & une & C.m & Tokombéré \\
\hline CM/EN/DW14 & Rouge pâle & Ovale & 28,5 & Sans contour du hile & une & C.m & Maroua, Mbé \\
\hline $\mathrm{CM} / \mathrm{AD} / \mathrm{CM} / 15$ & Marron & $\begin{array}{l}\text { Ovale et Bout } \\
\text { aplati }\end{array}$ & 26.75 & Sans contour du hile & $\begin{array}{c}\text { deux à } \\
\text { trois }\end{array}$ & C. $m$ & Tela, Meiganga, tignère \\
\hline $\mathrm{CM} / \mathrm{AD} / \mathrm{CM} / 16$ & Marron rouge & $\begin{array}{l}\text { Ovale et Bout } \\
\text { aplati }\end{array}$ & 44.5 & Sans contour du hile & $\begin{array}{c}\text { deux à } \\
\text { trois }\end{array}$ & C. $s$ & Tibati, Garga-limbona \\
\hline $\mathrm{CM} / \mathrm{AD} / \mathrm{CM} / 17$ & Marron foncé & $\begin{array}{l}\text { Ovale et bout } \\
\text { aplati }\end{array}$ & 31.5 & Sans contour du hile & deux & C. $s$ & Meidjamba , Ngaoundéré, Tala \\
\hline $\mathrm{CM} / \mathrm{AD} / \mathrm{CM} / 18$ & Marron claire & Ovale & 51 & Sans contour du hile & une & C. $s$ & Garga-limbona, Nyambaka \\
\hline CM/EN/DW/19 & Rouge bordeaux & $\begin{array}{c}\text { Ronde au } \\
\text { bout pointu }\end{array}$ & 27 & Sans contour du hile & une & C. $m$ & Maroua, Guidiguis, Kaélé \\
\hline
\end{tabular}




\begin{tabular}{|c|c|c|c|c|c|c|c|}
\hline $\mathrm{CM} / \mathrm{EN} / \mathrm{DW} / 20$ & Rouge taché de blanc & ovale & 52,5 & $\begin{array}{l}\text { Gris de forme } \\
\text { papillon }\end{array}$ & une & C.m & Gazawa, Tcholiré, Bogo \\
\hline CM/EN/DW/21 & Marron taché de noire & Ronde & 39,25 & Sans contour & Un/deux & C.m & $\begin{array}{l}\text { Mora, Guidiguis, Mindif, } \\
\text { Nyamboya }\end{array}$ \\
\hline $\mathrm{CM} / \mathrm{EN} / \mathrm{DW} / 22$ & Rouge sombre taché de blanc & Ronde & 43,5 & $\begin{array}{l}\text { Blanc de forme } \\
\text { papillon }\end{array}$ & une & $C . m$ & Guidiguis, Touloum \\
\hline $\mathrm{CM} / \mathrm{EN} / \mathrm{DW} / 23$ & tache noire sur fond marron & Ronde & 51,5 & Sans contour & une & $C . m$ & Mora , guidiguis \\
\hline CM/EN/DW/24 & Noir pointille sur fond blanc & ovale & 45 & Blanc circulaire & une & C.m & Moutourwa, Garoua \\
\hline $\mathrm{CM} / \mathrm{AD} / \mathrm{CM} / 25$ & Noir violet & Ronde & 30 & Noir irrégulier & une & C.m/C.s & Meiganga, Ngaoundéré \\
\hline $\mathrm{CM} / \mathrm{AD} / \mathrm{CM} / 26$ & Noir rayure & Ronde & 25,25 & Noir irrégulier & une & C.m & Meiganga, Ngaoundal \\
\hline $\mathrm{CM} / \mathrm{EN} / \mathrm{DW} / 27$ & Violet noire taché de blanc & Ronde & 50,75 & Sans contour & une & C.m/C.s & Moutourwa, Mbé \\
\hline CM/EN/DW/28 & Noire graines et rayure & Ronde & 45 & Sans contour du hile & une & C. $m$ & Guidiguis ,Mindif, Meiganga \\
\hline $\mathrm{CM} / \mathrm{AD} / \mathrm{MC} / 29$ & Tache violet sur fond blanc & Ovale & 49.4 & $\begin{array}{l}\text { Contour du hile gris } \\
\text { en forme de papillon }\end{array}$ & une & C. $m$ & Ngaoundal, Mayo-Darlé \\
\hline $\mathrm{CM} / \mathrm{EN} / \mathrm{MC} / 30$ & Tache noire sur fond brun & ronde et Aplati & 37.69 & Sans contour du hile & Une/deux & C. $m$ & Dziguilao, Touloum \\
\hline $\mathrm{CM} / \mathrm{AD} / \mathrm{MC} / 31$ & Gris pâle & $\begin{array}{c}\text { Ronde et bout } \\
\text { plat }\end{array}$ & 41.34 & Sans contour du hile & Une/deux & C. $s$ & Ngaoundal, Garoua, Djola \\
\hline $\mathrm{CM} / \mathrm{AD} / \mathrm{MC} / 32$ & Beige crème & ovale & 37.02 & $\begin{array}{l}\text { Contour du hile violet } \\
\text { en forme papillon }\end{array}$ & une & C. $s$ & Nyamboya, \\
\hline $\mathrm{CM} / \mathrm{EN} / \mathrm{MC} / 33$ & Rouge taché de noire & Ovale & 41.21 & Sans contour de hile & une & C. $m$ & Kaélé \\
\hline $\mathrm{CM} / \mathrm{EN} / \mathrm{MC} / 34$ & Tache grise en fond blanc & Ovale & 47.15 & Contour du hile violet & une & C. $m$ & Mbaiboum \\
\hline $\mathrm{CM} / \mathrm{EN} / \mathrm{MC} / 35$ & Noir au s'extrémité blanche & ovale & 36.13 & contour de hile noir & une & C. $m$ & Touboro, Maroua, Garoua \\
\hline $\mathrm{CM} / \mathrm{EN} / \mathrm{MC} / 36$ & Violet taché de blanc & ovale & 45 & Contour du hile brun & une & & \\
\hline $\mathrm{CM} / \mathrm{EN} / \mathrm{MC} / 37$ & $\begin{array}{l}\text { Rouge taché de noir au } \\
\text { s'extrémité blanche }\end{array}$ & ovale & 44.46 & Contour du hile beige & une & C. $m / C . s$ & Mbaiboum \\
\hline $\mathrm{CM} / \mathrm{EN} / \mathrm{MC} / 38$ & Rouge de rayure blanche & ovale & 50,6 & $\begin{array}{c}\text { Contour du hile } \\
\text { beige }\end{array}$ & une & C. $m$ & Touboro \\
\hline $\mathrm{CM} / \mathrm{EN} / \mathrm{MC} / 39$ & Blanche violet et rayure de noir & ovale & 51.44 & $\begin{array}{c}\text { Contour du hile blanc } \\
\text { et sous forme de } \\
\text { cercle }\end{array}$ & une & C. $m$ & Doukoula \\
\hline $\mathrm{CM} / \mathrm{EN} / \mathrm{MC} / 40$ & Blanc taché de rouge & ovale & 35.61 & Contour du hile gris & une & C. $m$ & Kaélé, Ngong, yagoua Mora \\
\hline $\mathrm{CM} / \mathrm{EN} / \mathrm{MC} / 41$ & Rouge uniforme & ovale & 42.35 & Sans contour du hile & une & C. $m$ & Kaélé \\
\hline CM/EN/MC/42 & Rouge au s'extrémité blanche & $\begin{array}{l}\text { ovale et } \\
\text { allongée }\end{array}$ & 44.42 & Contour du hile rouge & une & C. $m / C . s$ & Mbaiboum \\
\hline
\end{tabular}




\begin{tabular}{|c|c|c|c|c|c|c|c|}
\hline $\mathrm{CM} / \mathrm{EN} / \mathrm{MC} / 43$ & Blanc rayure noire & ovale & 41.05 & Contour du hile gris & une & C. $m$ & Touboro \\
\hline CM/EN/MC/44 & Beige rayure noire & ovale & 46.48 & $\begin{array}{l}\text { Contour du hile gris } \\
\text { en forme de papillon }\end{array}$ & une & C. $m$ & Touboro \\
\hline $\mathrm{CM} / \mathrm{EN} / \mathrm{MC} / 45$ & Blanc tachée du rouge bordeaux & ovale & 40.76 & Contour du hile gris & une & C. $m$ & Touboro \\
\hline $\mathrm{CM} / \mathrm{EN} / \mathrm{MC} / 46$ & Gris pointillé du rouge & $\begin{array}{l}\text { Ovale et un } \\
\text { peu arrondie }\end{array}$ & 49.17 & Contour du hile gris & & C. $m$ & Dziguilao, Mindif \\
\hline $\mathrm{CM} / \mathrm{EN} / \mathrm{MC} / 47$ & Kaki au s'extrémité blanche & ovale & 34.98 & $\begin{array}{l}\text { Contour du hile } \\
\text { marron }\end{array}$ & une & C. $m$ & Mbaiboum, \\
\hline $\mathrm{CM} / \mathrm{EN} / \mathrm{MC} / 48$ & Blanc violet & ovale & 43.11 & Contour du hile noir & une & C. $m$ & Maroua, kotek \\
\hline CM/EN/MC/49 & Blanc violet & ovale & 46.85 & Contour du hile gris & une & C. $m / C . s$ & Doukoula, Touloum \\
\hline $\mathrm{CM} / \mathrm{EN} / \mathrm{MC} / 50$ & Pointillé rouge sur fond blanc & ovale & 28.35 & $\begin{array}{l}\text { Contour du hile gris } \\
\text { en forme de papillon }\end{array}$ & une & C. $m$ & Doukoula, Dziguilao \\
\hline $\mathrm{CM} / \mathrm{EN} / \mathrm{MC} / 51$ & Rouge sombre & ovale & 32.4 & Sans contour du hile & une & C. $m$ & Gazawa, Salak \\
\hline $\mathrm{CM} / \mathrm{EN} / \mathrm{MC} / 52$ & Violet blanc taché de noire & ovale & 33.33 & Sans contour du hile & une & C. $m$ & Touloum, kaélé, Mindif \\
\hline $\mathrm{CM} / \mathrm{EN} / \mathrm{MC} / 53$ & Rouge taché de noire & ovale & 35.62 & Sans contour du hile & une & C. $m$ & Touloum, Mora \\
\hline $\mathrm{CM} / \mathrm{EN} / \mathrm{MC} / 54$ & Tache rouge sur fond blanc & ovale & 45.46 & Contour du hile rouge & une & C. $m$ & Maroua, Moutourwa \\
\hline
\end{tabular}

* les deux principaux ravageurs sont $: \mathrm{C} . \mathrm{m}=$ Callosobruchus maculatus et $\mathrm{C} . \mathrm{s}=$ Callosobruchus subinnotatus 
Les graines sont disponibles sur le marché à partir des mois d'avril et de mai dans la région de l'Adamaoua. Dans les régions du Nord et l'Extrême-Nord, les grains de voandzou sont présents durant toute l'année, avec une abondance maximale entre juin et juillet. Les semis sont réalisés sur des parcelles sillonnées, labourées ou par semis-direct à partir du mois de juillet pour les régions du Nord et l'extrême-Nord et début août pour la région de l'Adamaoua. Pour ce qui est de cette région, certaines paysannes ajoutent quelques pieds de maïs ou de boutures de manioc dans les parcelles de voandzou.

Le sarclage des parcelles de voandzou est pratiqué deux fois au moins avant la récolte. Aucun fertilisant n'est utilisé pour amender les sols car la plante a la capacité de fixer l'azote à travers ses racines, favorisant ainsi la reconstitution des nutriments $\mathrm{du}$ sol. La récolte dépend du cycle du morphotype semé au départ, et se fait pour la plus part à partir du mois de novembre. Les gousses sont récoltées à la main pour les sols meubles et avec une houe pour des sols compacts. Les gousses sont ensuite séchées sur des nattes en plein soleil, de préférence dans un endroit le mieux ventilé possible. Après la phase de séchage suit le stockage de la denrée dans divers modules.

\section{Usages des graines de voandzou dans la zone d'étude}

Les graines de voandzou sont utilisées aussi bien en alimentation humaine que pour le traitement de certaines pathologies. Comme aliment, les graines sont mangées crues ou cuites fraiches ou séchées. Les graines crues sont grillées ou rôties à la vapeur ou en ragoût. Quelques fois les graines sont réduites en farine et introduites seules ou de façons combinées dans les préparations des repas ou dans la préparation des beignets. Comme médicament, les graines remédient aux troubles digestifs, nerveux et aux courbatures.

\section{Module et forme de stockage du voandzou adopté par les paysans du Cameroun septentrional}

Les graines de voandzou après récolte sont stockées dans divers endroits dans l'habitation des producteurs. Dans la région de l'Extrême-Nord où les productions sont les plus importantes, la conservation se fait dans des greniers construits à part dans la concession familiale. En général, dans l'espace d'étude, les graines de voandzou sont stockées dans les magasins, les cuisines où les hangars. Les magasins de stockage individuel ou collectif restent les lieux où les graines de voandzou sont les plus conservés. Avant le stockage, les graines sèchent sur des «danki » ou tables à séchage et rarement en champ.

Les graines de voandzou récoltées sont stockées sous deux formes, en gousse et sans gousse avec une durée de stockage compris entre 6 et 9 mois 
en moyenne selon la zone. Dans la région de l'Adamaoua, l'intervalle entre la mise en stock du voandzou et la sortie des semences est de $6 \pm 1$ mois du fait de la saison sèche moins longue. Dans le Nord et l'Extrême-Nord, le stockage de la réserve dure $9 \pm 1$ mois compte tenu du retour tardif des pluies. De même, $69,33 \%$ des productrices du voandzou conservent les graines avec la gousse, contre $30,66 \%$ sans gousse. Elles estiment que les graines sont mieux protégées avec la gousse, qui est assimilée à une barrière de protection car la graine nue est très vulnérable aux attaques. Par contre dans l'Extrême-Nord, le stockage sans gousse est le plus pratiqué avec 56\% contre $44 \%$. Quant à la région du Nord, $60 \%$ conservent avec gousse et $40 \%$ sans gousse. Les paysannes du Nord apprécient de bien étaler leurs graines en gousse sur une surface aménagée sur la cour à ciel ouvert ou le soleil peut de manière continue faire sécher les graines. Dans l'Extrême-Nord et le Nord, 8 mois est la durée de conservation d'un bon nombre de productrices, l'exception de quelquesunes qui vont jusqu'à 9 mois espérant une flambée des prix des denrées qui coïncide avec la période de soudure où les greniers sont vides.

\section{Inventaire des insectes inféodés aux stocks de voandzou dans le Cameroun septentrional}

Les enquêtes montrent que les coléoptères des familles des Chrysomelidae sont les insectes les plus rencontrés dans les stocks de voandzou dans les trois régions. Les attaques commencent dans le champ et se poursuivent dans les modules. Les infestations des gousses par les déprédateurs ont lieu en cours de culture, mais le développement des larves dans les cotylédons des graines se poursuit pendant la phase de stockage (Glitho, 1990; Ouédraogo et al., 1996; Sanon et al., 2006). Ainsi, les potentiels destructeurs de voandzou en stock, sont principalement Callosobruchus maculatus et $C$. subinnotatus, avec une dominance de $C$. maculatus qui représente 99,67\% des ravageurs. En effet, les précédentes enquêtes menées dans cette zone d'étude, ont révélé que $C$. maculatus et $C$. subinnotatus sont les plus fréquemment rencontrés dans les stocks paysans (Ngamo et al., 2016). Face à l'étendue de leurs attaques et les conséquences sur la qualité du voandzou, ces déprédateurs constituent sans doute une des contraintes majeures au développement des cultures de légumineuses à graines et dont le voandzou.

Ces bruches qui ravagent le voandzou au cours du stockage sont parasitées par trois familles de parasitoïde : Trichogrammatidae, Eupelmidae, Pteromalidae (Luca, 1979; Van Huis 1991). La diversité des ennemis naturels des bruches dans les bassins de production dans l'Extrême- Nord du Cameroun comprend deux espèces appartenant à la famille de Pteromalidae (Annisopterromalus calandare et Dinarmus basalis) et un Eupelmidae 
(Eupelmus vuilleti). D. basalis est l'espèce la plus présente avec une proportion de 79,26\% des parasitoïdes récoltés (Tableau 3).

Tableau 3 : Biodiversité des ravageurs du voandzou et de leurs parasitoïdes extraits des stocks de voandzou collectés dans les trois régions septentrionales du Cameroun (2015 à

2017) et observés sur un total de 250 échantillons pendant trois mois au laboratoire.

\begin{tabular}{|c|c|c|c|c|c|c|}
\hline & Nom & 2015 & 2016 & 2017 & Total & $\%$ \\
\hline 1 & Tribolium castaneum(Herbst) & 12 & 29 & 47 & 88 & 0,1 \\
\hline 2 & $\begin{array}{l}\text { Callosobruchus maculatus } \\
\text { (Fab.) }\end{array}$ & 1082 & 3168 & 79041 & 83291 & 99,67 \\
\hline \multirow[t]{2}{*}{3} & $\begin{array}{l}\text { Callosobruchus subinnotatus } \\
\text { (Pic.) }\end{array}$ & 137 & 10 & 38 & 185 & 0,22 \\
\hline & Total ravageurs & 1231 & 3207 & 79126 & 83564 & 100 \\
\hline 4 & $\begin{array}{l}\text { Anisopteromalus calandrae } \\
\text { (HOW) }\end{array}$ & 0 & 153 & 74 & 227 & 3,54 \\
\hline 5 & Dinarmus basalis (Rondani) & 911 & 2056 & 2111 & 5078 & 79,26 \\
\hline \multirow[t]{2}{*}{6} & Eupelmus vuilleti (Crawf) & 55 & 190 & 857 & 1102 & 17,2 \\
\hline & Total parasitoïdes & 966 & 2399 & 3042 & 6407 & 100 \\
\hline
\end{tabular}

\section{Méthodes usuelles de protection des graines de voandzou au cours du stockage}

Les producteurs utilisent divers outils pour la protection du voandzou contre les bruches, principaux insectes nuisibles durant le stockage. Dans le Nord Cameroun quatre principales méthodes de protection sont identifiées : l'utilisation des pesticides chimiques industriels (43\%), l'utilisation des produits végétaux (28\%), de produits inertes dont principalement les cendres (20\%) et divers autres outils $(8,7 \%)$. L'utilisation des produits chimiques est la méthode la plus répandue et les insecticides utilisés sont en général dangereux. Le tableau 4 montre que plus de $50 \%$ de la population dans chacune de ces trois régions restent attachés à l'utilisation des produits synthétiques. Cependant la proportion de l'Extrême-Nord (62\%) est encore plus grande, dû au fait que l'accès à ces produits illicites sont sans obstacles dans le marché, ils proviennent de la contrebande au niveau des frontières voisines. Pour ce qui est des plantes introduites dans les modules, certaines utilisent une plante seule et d'autres des combinaisons allant de deux à quatre plantes dans le but d'obtenir une plus grande efficacité (Tableau 4).

Tableau 4 : Combinaisons de plantes introduites dans les stocks de voandzou par les producteurs (Pr : Nombre de producteurs concerné) pour réduire les attaques des ravageurs au cours du stockage

\begin{tabular}{|l|l|l|}
\hline Plantes combinées & $\begin{array}{l}\text { P } \\
\mathrm{r}\end{array}$ & $\begin{array}{l}\text { Marchés de denrées stockées } \\
\text { prospectés }\end{array}$ \\
\hline Combinaisons de deux plantes & & \\
\hline Capsicum frutescens + Allium sativum & 6 & Lara, Mindif, Yagoua, Doukoula, \\
\hline Capsicum frutescens + Annona senegalensis & 5 & Tchaklina, Wina, Kalfou \\
\hline Eucalyptus saligna + Annona senegalensis & 0 & Yagoua, Kai-kai \\
& 2 & \\
\hline
\end{tabular}




\begin{tabular}{|l|c|l|}
\hline Capsicum frutescens + Azadirachta indica & 8 & $\begin{array}{l}\text { Mindif, Kaélé, Moutourwa } \\
\text { Guidiguis, Lara, Touloum }\end{array}$ \\
\hline $\begin{array}{l}\text { Annona senegalensis }+ \text { Piliostigma } \\
\text { reticulatum }\end{array}$ & 0 & Tcherfeke, hounou,Dabaye \\
\hline Hyptis spicigera + Azadirachta indica & 4 & \\
\hline Hyptis spicigera + Capsicum frutescens & 0 & $\begin{array}{l}\text { Mindif, Kaélé, Moutourwa, } \\
\text { Doumrou, Guidiguis, Doukoula }\end{array}$ \\
\hline Hyptis spicigera + Dalbergia odorifera & 7 & $\begin{array}{l}\text { Moutourwa, Guidiguis, Touloum, } \\
\text { Yagoua }\end{array}$ \\
\hline $\begin{array}{l}\text { Ocimum canum }+ \text { Hyptis spicigera } \\
\text { Ocimum canum }+ \text { Capsicum frutescens }\end{array}$ & 3 & Tchaklina, Dana \\
\hline $\begin{array}{l}\text { Ocimum canum }+ \text { Allium sativum } \\
\text { Ocimum }\end{array}$ & 6 & $\begin{array}{l}\text { Moutourwa, Doumrou, Guidiguis, } \\
\text { Vada, }\end{array}$ \\
\hline Combinaisons de trois plantes & 5 & Mindif, Guidiguis, Yagoua \\
\hline $\begin{array}{l}\text { Azadirachta indica }+ \text { Hyptis spicigera }+ \text { Vepris } \\
\text { heterophylla }\end{array}$ & 3 & Lara, Mindif, Hounou, \\
\hline $\begin{array}{l}\text { Azadirachta indica }+ \text { Hyptis spicigera }+ \\
\text { Capsicum frutescens }\end{array}$ & 9 & $\begin{array}{l}\text { Mindif, Kaélé, } \\
\text { Lara, Touloum }\end{array}$ \\
\hline $\begin{array}{l}\text { Azadirachta indica }+ \text { Cpasicum frutescens }+ \\
\text { Sorghum bicolor }\end{array}$ & 5 & Mindif, Kaélé, Moutourwa, Lara, \\
\hline $\begin{array}{l}\text { Allium sativum }+ \text { Capsicum frutescens }+ \text { Allium } \\
\text { sp }\end{array}$ & 2 & Lara \\
\hline $\begin{array}{l}\text { Azadirachta indica }+ \text { Hyptis spicigera }+ \\
\text { Nicotina tabacum }\end{array}$ & 1 & Loum, Yagoua, \\
\hline $\begin{array}{l}\text { Azadirachta indica }+ \text { Hyptis spicigera }+ \\
\text { Ocimum canum }\end{array}$ & 1 & Touloum \\
\hline Combinaisons de quatre plantes & & \\
\hline $\begin{array}{l}\text { Aroundo donax }+ \text { Ficus abitulifolia }+ \text { Vepris } \\
\text { heterophylla }+ \text { Teramnus labialus }\end{array}$ & 1 & Moutourwa \\
\hline $\begin{array}{l}\text { Aroundo donax }+ \text { Ficus abitulifolia }+ \text { Vepris } \\
\text { heterophylla }+ \text { Capsicum frutescens }\end{array}$ & 3 & Moutourwa, Mindif. \\
\hline
\end{tabular}

Les insecticides sont les produits chimiques industriels les plus utilisés dans le traitement post-récolte des graines de voandzou. Les produits de fumigation à base de phosphure d'aluminium comme le PHOSTOXIN le JUSTOXIN, et à base de dichlorvos comme le INSCET KILLER connu localement sous le nom «PIAPIA», sont les plus populaires. Le phosphure d'aluminium est classé d'après l'homologation camerounaise comme substance extrêmement dangereuse mais qui est malheureusement homologuée (MINADER, 2015). Le dichlorvos quant à lui n'est pas homologué au Cameroun, mais sur la liste des produits phytosanitaires identifiées par l'OMS (2009) est une matière active extrêmement dangereuse classée Ia (Tableau 5). 
Tableau 5 : Liste des pesticides utilisés pour le traitement des denrées stockées et leur popularité, leur licité et leur dangerosité

\begin{tabular}{|c|c|c|c|c|c|}
\hline & Nom Commercial & $\begin{array}{l}\text { Homologati } \\
\text { on }\end{array}$ & Matière active & $\mathrm{Cl}^{*}$ & Effectif \\
\hline 1 & $\begin{array}{l}\text { CONQUEST C } \\
176 \mathrm{EC}\end{array}$ & non & $\begin{array}{l}\text { acétamipride + } \\
\text { cyperméthrine }\end{array}$ & $\begin{array}{l}\text { II+ } \\
\text { III }\end{array}$ & 1 \\
\hline 2 & CYPERCAL 720EC & 605/11/IN & cyperméthrine & II & 2 \\
\hline 3 & $\begin{array}{l}\text { INSECT KILLER } \\
\text { (PIAPIA) }\end{array}$ & non & dichlorvos & Ia & 7 \\
\hline 4 & INSECTOR & 430/08/IN & imidaclopride & II & 4 \\
\hline 5 & JUSTOXIN & non & phosphure d'aluminium & Ia & 15 \\
\hline 6 & CALTIO & 683/11/IN & thirame+ chlorpyriphos & $\begin{array}{l}\text { III+I } \\
\text { I }\end{array}$ & 4 \\
\hline 7 & POUDROX & 492/09/IN & malathion & III & 5 \\
\hline 8 & PUSH OUT & non & phosphide de zinc & indét & 3 \\
\hline 9 & RATICIDE & non & brodifacoum & Ia & 1 \\
\hline 10 & STARGRAIN 2DP & $515 / 09 / \mathrm{IN}$ & deltaméthrine & II & 4 \\
\hline 11 & TERMITOX & non & lindane & POP & 2 \\
\hline 12 & PROTECT DP & 769/13/IN & $\begin{array}{l}\text { deltaméthrine+ } \\
\text { pyrimophos-methyl }\end{array}$ & $\begin{array}{l}\text { II+II } \\
\text { I }\end{array}$ & 4 \\
\hline 13 & MOMTAZ 45WS & $\begin{array}{l}\text { 419/07/INF } \\
\text { O }\end{array}$ & imidaclopride+thirame & II+II & 6 \\
\hline 14 & FORCETOXIN & non & aluminum phosphide & indet & 10 \\
\hline 15 & PERFECT KILLER & non & chlorpyriphos & II & 3 \\
\hline 16 & RAMBO & 0842/A0/In & transfluthrine & III & 1 \\
\hline 17 & MAMIRA SUPER & non & imidachlopride & II & 1 \\
\hline 18 & OPTIMAL SUPER & 0694/AI/In & acetamipride & III & 2 \\
\hline 19 & FURABAK & non & carbofuran & $\mathrm{Ib}$ & 1 \\
\hline 20 & MORAN 30DP & 0640/AI/In & indoxacarbe & III & 1 \\
\hline 21 & EXPERT & non & emamectine benzoate & III & 2 \\
\hline 22 & VERTOX & 0691/AI/Ro & brodifacoum & Ia & 1 \\
\hline 23 & RODILON & non & difethialone & Ia & 1 \\
\hline 24 & SUPER 12EC & non & cypermetrine & II & 2 \\
\hline 25 & NO FEAR & non & chlorpyriphos & II & 1 \\
\hline
\end{tabular}

Effectif $=$ nombre de personne utilisant la substance dans un échantillon de producteur

* Classification de l'OMS : Ia= extrêmement dangereux ; II = très dangereux ; III = modérément dangereuxCeci nous amène à penser que les denrées vendues dans ces marchés sont pour la plus part de qualités douteuses, étant donné que ces produits sont généralement appliqués sur les grandes quantités de voandzou conservées dans des sacs qui seront par la suite détaillées aux petites vendeuses.

\section{Conclusion}

Dans les Régions septentrionales du Cameroun, les productrices ont un grand choix de morphotypes de voandzou à cultiver. Malgré le potentiel que cette culture a du fait de ses vertus, les productions évoluent peu. Le principal danger à la perte de la qualité et même de la quantité des productions est la pression des insectes ravageurs. Le risque d'intoxication dû à l'utilisation des pesticides chimiques dangereux est réel, il y a donc une nécessité à développer et à disséminer des formulations insecticides à bases des plantes locales 
surtout des plantes à épices déjà consommées par les populations pour limiter les pertes au cours du stockage. De ce fait les fruits des espèces telles que Piper nigrum Linée. et de Syzygium aromaticum (L.) Merr. et L. M. Perry., sont utilisés comme épice en cuisine et aussi pour le stockage des grains. De par les arômes qu'elles libèrent, éloignent les insectes ravageurs qui viennent et tuent ceux qui restent dans les structures de stockage et empêchent de nouvelles infestations.

La promotion de ces essences condimenteuses, peut se faire par une maîtrise de leur utilisation et par leur vulgarisation. Etant donné que cette recherche des méthodes alternatives de protection, propose des solutions peu coûteuses qui respectent l'homme et l'environnement.

\section{References:}

1. Delobel A, Tran M. (1993). Les Coléoptères des denrées alimentaires entreposées dans les régions chaudes. ORSTOM/CTA. Faune tropicale $424 \mathrm{p}$.

2. FAO

http://countrystat.org/home.aspx?c=CMR\&ta=032CPD010\&tr=7.

3. Fotso, pasquet, (1991). Les légumineuses alimentaires du Cameroun. Centre de Nutrition, IMPM-BP 6163 - Yaoundé, Cameroun, 346 p.

4. Glitho I.A. (1990). Les Bruchidae ravageurs de Vigna unguiculata (WaIp) en zone guinéenne. Analyse de la diapause reproductrice chez les mâles de B. atrolineatus Pic. Thèse de Doctorat, Tours. 100 p.

5. IPGRI/IITA/BAMNET. (2000). Descriptors for bambara groundnut: 48, Rome, Italy

6. Luca De Y. (1979). Ingrédients naturels de préservation des grains stockés dans les pays en voie de développement. Journal d'agriculture traditionnelle et de botanique appliquée : 26(1) : 29-52

7. Manetsa V. (2011). Etude multi-échelles des précipitations et du couvert végétal au Cameroun : analyses spatiales, tendances temporelles, facteurs climatiques et anthropiques de variabilité du NDVI. Sciences de la Terre. Université de Bourgogne.

8. Minader, (2015). Index Phytosanitaire du Cameroun, édition 2014. Ministère de l'Agriculture et du Développement Rural Ed. Yaoundé 395 pp.

9. Nacoulma-Ouédraogo O. (1996). Plantes médecinales et pratiques traditionnelles au Burkina-Faso : cas du plateau central. Thèse de Doctorat ès Sciences Naturelles, Université de Ouagadougou. Ouagadougou, Burkina Faso.

10. Ngamo TLS, Goudoum A, Djakissam W, Madou C. (2016). Les bruches du voandzou Vigna subterranea (L.) et les outils de protection 
post récolte dans le Nord du Cameroun. Entomologie Faunistique Faunistic Entomology 2016 69, 83-89 PP.

11. Onwubiko N.I.C., Odum O.B., Utazi C.O. And Poly-Mbah P.C. (2011). Studies on the adaptation of Bambara Groundnut [Vigna Subterranea (L.)Verdc] in Owerri Southeastern Nigeria. New York Science Journal, 4(2):60-67.

12. Ouedraogo AP, Sou S, Sanon A, Monge JP, Huignard J, Tran B. (1996 b). Influence of temperature and humidity on populations of Callosobruchus maculatus Fab. (Coleoptera : Bruchidae) and its parasitoid Dinarmus basalis Rond. (Pteromalidae) in two climatic zones of Burkina Faso. Bul. Of Entomo/. Res.. 86, 695-702.

13. Sanon, A., Ilboudo, Z., Dabiré, L. C. B., Nebie, R. C. H., Dicko, I. O., \& Monge, J. P., (2006). Effects of Hyptis spicigera Lam. (Labiatae) on the behaviour and development of Callosobruchus maculatus F. (Coleoptera: Bruchidae), a pest of stored cowpeas. International Journal of Pest Management, 52, 117 - 123.

14. Stephens J.M. (2009). Bambara Nut (Voandzeia subterranea (L.) Thouars.) Horticultural Sciences Department, Florida Cooperative Extension Service, Institute of Food and Agricultural Sciences, University of Florida, HS547, pp: 1-2. Retrieved from: http://edis.ifas.ufl.edu.

15. Van huis A. (1991). Biological methods of bruchid control in the tropics: a review. Insect Science and ifs Application. Vol. 12, $\mathrm{N}^{\circ} 1 / 2 / 3$, pp. 87-102.

16. Who, (2009). The WHO recommended classification of Pesticides by hazard and guidelines to classification. International Program on Chemical Safety. 77pp.

17. Yusuf A. A., Ayedun,H., \& Sanni L. O. (2008). Chemical composition and functional properties of raw and roasted Nigerian benniseed (Sesamum indicum) and bambara groundnut (Vigna subterranean). Food Chemistry. 111: 277-282. http://dx.doi.org/10.1016/j.foodchem.2007.12.014 